\title{
Cosmic Ringtones in Pulsar Data?
}

\author{
A pulsar survey has detected a potential signal from low-frequency \\ gravitational waves, which theorists are eager to explain.
}

\section{By Michael Schirber}

magine a gravitational-wave detector stretching over a sizeable chunk of our Galaxy. That, in a nutshell, is the North American Nanohertz Observatory for Gravitational Waves (NANOGrav), which monitors distances in our cosmic neighborhood using a network of clock-like stars, called pulsars. In late 2020, the NANOGrav team reported seeing fluctuations in the timing of pulsar ticks, which could be evidence of gravitational waves at nanohertz frequencies [1].

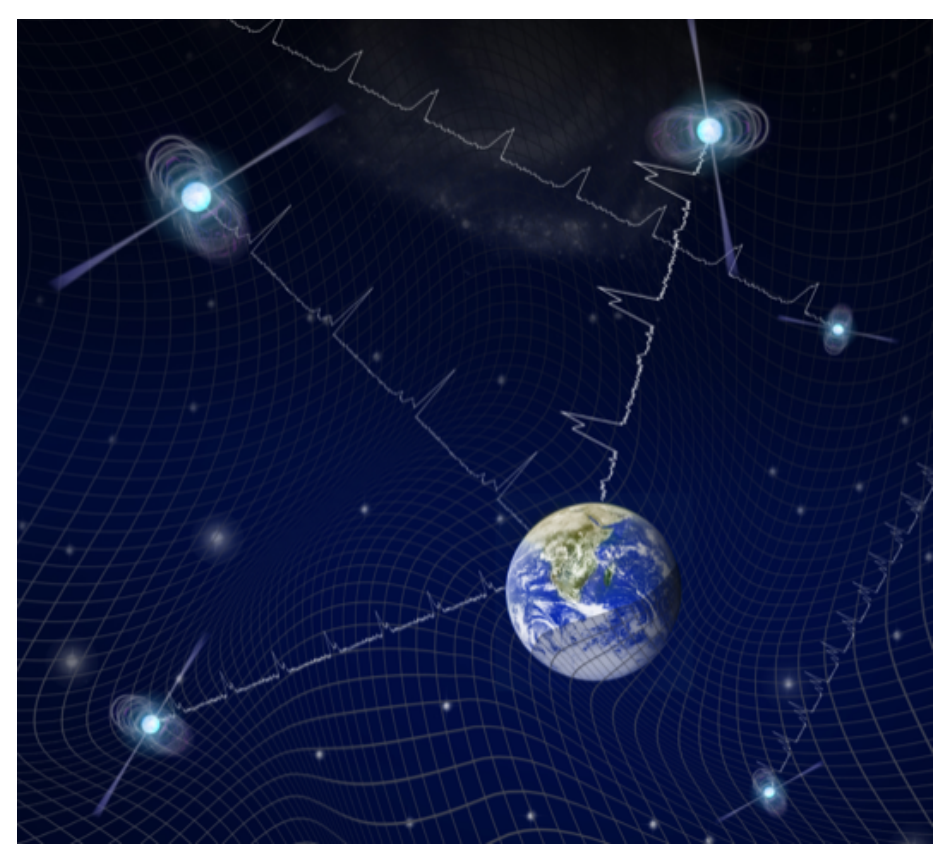

By monitoring the radio flashes from distant pulsars, astronomers have spotted a signal that could be the result of a background of gravitational waves.

Credit: Tonia Klein/NANOGrav
The source of such slow-cycling waves could be black hole mergers, but several new theoretical studies-all appearing in Physical Review Letters-propose that other, more exotic objects could explain the data. One possibility is that superdense filaments, called cosmic strings, produced the gravitational radiation through the vibrations of string-tied loops $[2,3]$. Another idea is that the waves originated during the birth of so-called primordial black holes, which are thought to have formed in the embers of the big bang $[4,5]$. If the NANOGrav signal does come from gravitational waves, then further pulsar observations within the next five years may be able to discriminate between these different source candidates.

Pulsars are rapidly rotating neutron stars emitting radio beams that sweep over Earth at regular intervals, like the light from a lighthouse. Their connection to gravitational waves goes back to 1974, when physicists Russell Hulse and Joseph Taylor explained the slowdown of a pulsar as a consequence of the emission of gravitational radiation. The direct detection of gravitational waves came in 2016, when the LIGO experiment uncovered the tiny, wave-induced changes in the lengths of its giant interferometer arms. NANOGrav looks for similar space-distorting effects in the 1000-light-year-distances to 45 pulsars spread over the sky. If a gravitational wave passes between Earth and one of the pulsars in the array, the distance change would affect the timing of the pulsar's radio flashes, which arrive roughly once every millisecond. "The kind of timing precision that you can get from a millisecond pulsar really rivals that of an atomic clock," says Joseph Simon of the NANOGrav Collaboration.

The 2020 NANOGrav result, based on the analysis of 12.5 years 
of data, shows timing fluctuations over multiyear timescales. These fluctuations happen in all the pulsars, which argues against this being some intrinsic noise in the pulsar emission. "There's no way for the pulsars to talk to each other to coordinate their behavior," Simon says. Instead, the common fluctuations could come from a random background-or "hum"-of gravitational waves. "The detection of a stochastic background in gravitational waves is one of the most anticipated and important milestones in gravitational-wave astronomy," says Kai Schmitz from CERN in Switzerland.

The NANOGrav results still leave room for doubt. "I'm confident that the signal itself is real," says Simon. But he admits that the gravitational-wave interpretation requires further evidence. In particular, gravitational waves should imprint a so-far-unseen correlation pattern among pulsars: the fluctuations of pulsars that are close to each other in the sky should be more correlated than those of angularly separated pulsars.

If the signal truly comes from gravitational waves, a natural explanation would involve black hole mergers. To generate waves at nanohertz frequencies, the merging black holes would have to weigh millions of times more than the solar-mass-scale black holes detected by LIGO and Virgo. Astronomers know that supermassive black holes inhabit the centers of most galaxies. Since galaxies merge, it's reasonable that supermassive black holes could also merge. But several uncertainties surround this scenario, Schmitz says, such as whether the black holes can form the tight orbiting binaries needed to generate gravitational waves.

Alternative gravitational-wave sources are possible. Schmitz and his colleagues show that the NANOGrav data can be reproduced by cosmic strings-dense, one-dimensional objects that are predicted to arise during phase transitions in the space-filling fields of the early Universe [2]. The strings would form a crisscrossing network throughout space. Over time, these strings would collide or intersect with themselves, creating closed loops that oscillate to produce gravitational waves. The models suggest that such loops would form throughout the history of the Universe, with the most recent loops stretching over light-year scales and producing low-frequency gravitational waves. "If you see a wave signal at nanohertz frequencies, a likely culprit would be cosmic strings," says Marek Lewicki from the University of Warsaw in Poland. He

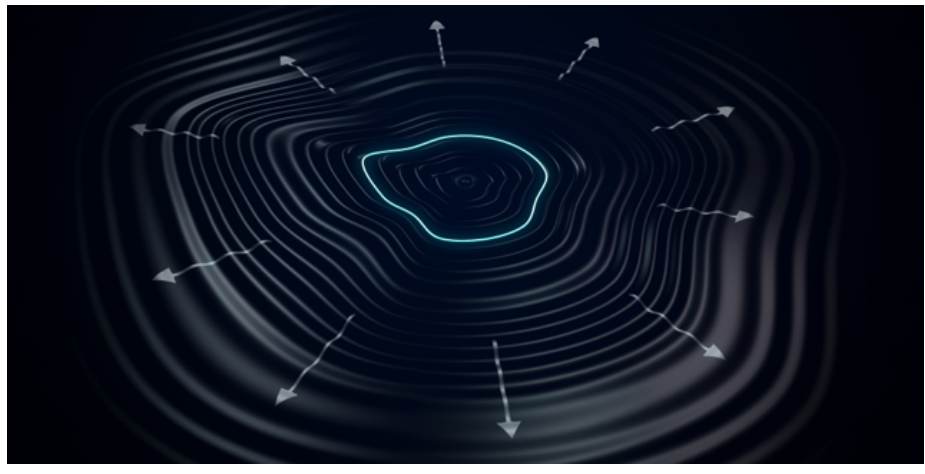

Cosmic strings that are closed into loops could produce gravitational waves at a wide range of frequencies, including those probed by NANOGrav.

Credit: Daniel Dominguez/CERN

and John Ellis from King's College London have also modeled the cosmic-string gravitational-wave background, delivering results consistent with those of Schmitz and collaborators [3].

Both models predict that cosmic strings would produce gravitational waves over a wide range of frequencies. This broad spectrum is distinct from the spectrum of supermassive black hole mergers, which is expected to fall off steeply toward millihertz frequencies. Lewicki says that scientists should be able to probe these higher-frequency regions with LISA, a space-based gravitational-wave detector scheduled to fly in 2034.

A third explanation involves primordial black holes. These hypothetical objects could have formed in the very early Universe in processes that would also generate gravitational radiation. The frequency of gravitational waves would be related to the black hole masses, explains Gabriele Franciolini from the University of Geneva. The nanohertz signal seen by NANOGrav corresponds to black holes in the 0.1-0.01 solar-mass range. Franciolini and other members of Antonio Riotto's group in Geneva have imagined a scenario in which primordial black holes are created over a wide range of masses, from solar to asteroid size [4]. An appealing aspect of this proposal is that it can account not only for NANOGrav's observations but also for dark matter. "There is a window where you can have $100 \%$ of the dark matter in the form of primordial black holes," Franciolini says. Other researchers 


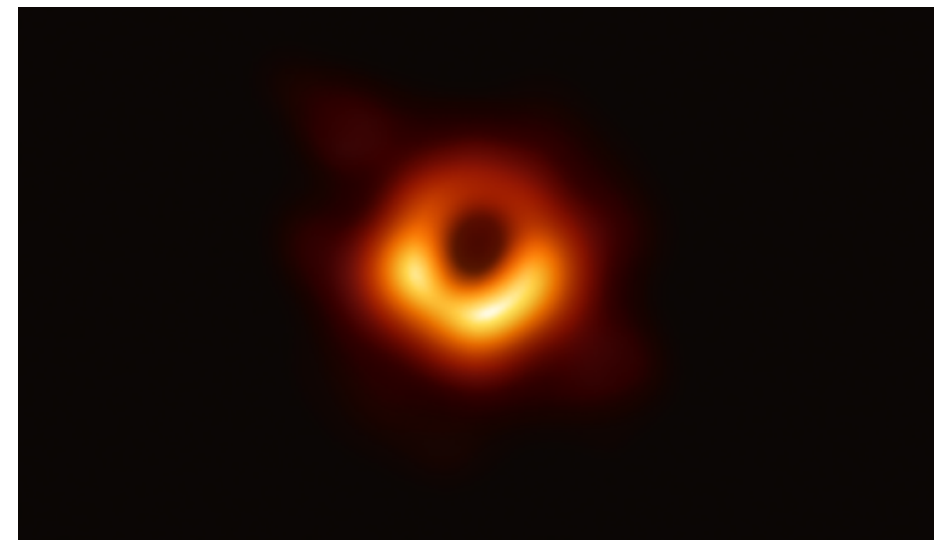

This image shows the supermassive black hole at the center of the nearby galaxy M87. Black holes of this size could generate nanohertz gravitational waves through their mergers. Other wave-generating scenarios consider primordial black holes that may have formed in the early Universe and could have been the seeds for supermassive black holes.

Credit: Event Horizon Telescope

based in Spain and Estonia have proposed a similar model but with primordial black holes in a higher mass range between a thousand and a million solar masses [5]. Such objects could not be the dark matter, but they might solve the puzzle of how supermassive black holes are formed.

"There's a lot of excitement right now because if the NANOGrav signal is a primordial one, we could constrain early Universe theories, as well as particle physics theories," Franciolini says. Characterizing the gravitational-wave spectrum could provide information about the Universe's expansion history and about particle physics at energies unattainable with current particle colliders. But the needed spectral measurements may be decades away.

In the short term, the NANOGrav team plans to verify whether its signal is real. The collaboration suffered a setback this past December, when the Arecibo radio telescope in Puerto Rico collapsed. "Arecibo made up roughly half of our sensitivity, so its loss is really impactful," Simon says. NANOGrav is working with international partners to find telescopes that can fill in for Arecibo. In the meantime, the team is working on a comparison of their data with other pulsar timing array projects in Europe and Australia. "You should expect a confirmation-or not-of the signal within the next six to nine months," Simon says. He expects that, if the signal is real, astronomers will have a clearer sense of the spectrum shape in the next five years, which could allow discrimination between different models.

"The future for pulsar timing astronomy is very bright," Schmitz says. If a young student asked for career advice, he would recommend looking into this field. "You will certainly see lots of exciting developments in the next five to ten years," he says.

Michael Schirber is a Corresponding Editor for Physics based in Lyon, France.

\section{REFERENCES}

1. Z. Arzoumanian et al., "The NANOGrav 12.5 yr data set: Search for an isotropic stochastic gravitational-wave background," Astrophys. J. Lett. 905, L34 (2020).

2. S. Blasi et al., "Has NANOGrav found first evidence for cosmic strings?" Phys. Rev. Lett. 126, 041305 (2021).

3. J. Ellis and M. Lewicki, "Cosmic string interpretation of NANOGrav pulsar timing data," Phys. Rev. Lett. 126, 041304 (2021).

4. V. De Luca et al., "NANOGrav data hints at primordial black holes as dark matter," Phys. Rev. Lett. 126, 041303 (2021).

5. V. Vaskonen and H. Veermäe, "Did NANOGrav see a signal from primordial black hole formation?” Phys. Rev. Lett. 126, 051303 (2021). 